\title{
In Search for Your Own Virtual Individual
}

\author{
L. Moccozet ${ }^{1}$, A. Garcia-Rojas ${ }^{2}$, F. Vexo ${ }^{2}$, D. Thalmann ${ }^{2}$, \\ and N. Magnenat-Thalmann ${ }^{1}$ \\ ${ }^{1}$ MIRALab, University of Geneva, Route de Drize, 7, Site de Battelle \\ CH1227 Carouge/Genève, Switzerland \\ \{moccozet, thalmann\} @miralab.unige.ch \\ ${ }^{2}$ VRLab, EPFL, Lausanne \\ CH-1215 Lausanne, Switzerland \\ \{alejandra.garciarojas, frederic.vexo, daniel.thalmann\}@epfl.ch
}

\begin{abstract}
The use of inhabited Virtual Environments is continuously growing. People can embody a human-like avatar to participate inside these Virtual Environments or they can have personalized character acting as mediator; sometimes they can even customize it to some extent. Those Virtual Characters belong to the software owner, but they could be potentially shared, exchanged and individualized between participants, such as already proposed by Sony with Station Exchange. Technology with standards could significantly improve the exchange, the reuse and the creation of such Virtual Characters. However an optimal reuse is only possible if the main components of the characters: geometry, morphology, animation and behavior, are annotated with semantics. This may allow to users searching for specific models and customize them. Moreover search technology based on the Web Ontology Language (OWL) can be implemented to provide this type of service. In this paper we present the considerations to build an ontology that fulfills the mentioned proposes.
\end{abstract}

Keywords: 3D shapes, Virtual Environments, Virtual Human, Standards, Ontology, Web Ontology Language.

\section{Introduction}

Complex 3D shapes such as Virtual Humans are becoming more and more popular in various areas. One of them is the game industry, where players are represented with human like avatars in immersed in a Virtual Environments.

On one hand, with the successful development of massively multiplayer online role playing games or MMORPG and their persistent virtual worlds, it becomes common to exchange or to buy $3 \mathrm{D}$ objects such as virtual weapons and tools or even 3D virtual human characters used as avatars. For example, Sony is proposing Station Exchange [1] (Fig. 1), to allow players exchanging virtual characters. On the other hand, the recent advent of efficient and accurate acquisition systems and reconstruction platforms has potentially increased the availability and dissemination of complex digital shapes such as Virtual Humans [2,3]. As a consequence, the supply and demand for Virtual Human will probably rapidly expand. However, the 
infrastructures that would be required for sharing large databases of Virtual Human models and populating Virtual Environments are still not mature; moreover a common understanding to share this models and their information does not exist.

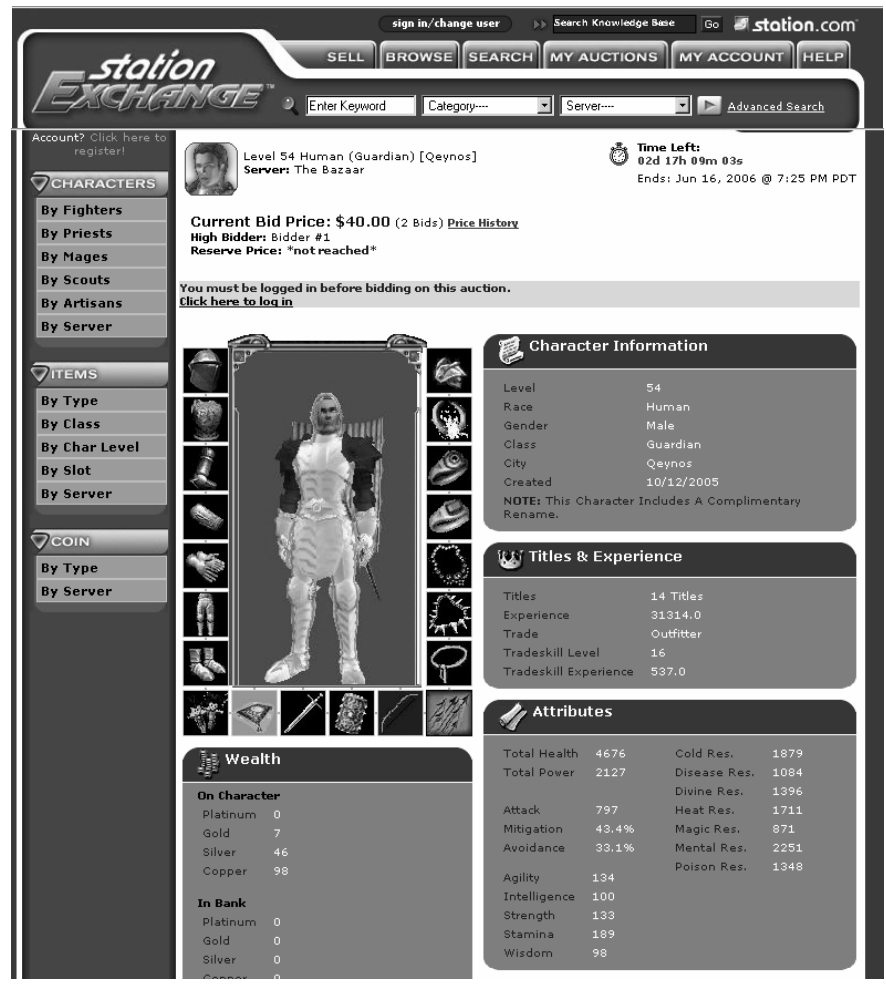

Fig. 1. Snapshot from a Virtual Character proposed for auction on the Sony Station Exchange web server

Considering a Virtual Environment, the individualization of character is possible, but it is limited to some body shapes and changes of skin and hears colors, garments, accessories, etc. a large variety is not accessible. This is because it is expensive for designers to create a large number of characters and accessories. As a consequence, we may consider other techniques for the creation of virtual humans such as scanning and example-based or knowledge-based methods [2, 3] (Fig. 2). Therefore we have more options for shape creation.

The conventional shape acquisition and processing pipeline severely neglects to manage and take advantage of the knowledge associated to shapes as it would be required to efficiently share and interoperate their digital representation. Moreover, digital shapes such as Virtual Humans include different layers of semantic information. A Virtual Human is usually considered to a limited extent as a 3D shape with control animation structures. This representation only counts for a physical representation and movement control of the Virtual Human. However Virtual Human models should not only provide a physical representation of Humans: they need to 
personify Humans as described by Kshirsagar et al. in [4]. Processing a 3D model of a human to its virtual similar can be seen as anthropomorphism or personification. Anthropomorphism ${ }^{1}$, also referred to as personification ${ }^{2}$ or prosopopoeia, means to concede human attributes to inanimate objects. These attributes may include sensations, emotions, desires, physical gestures and expressions, and powers of speech, among others. In our context it consists in representing a virtual counterpart of a real or "potential" Human from a virtual 3D shape.

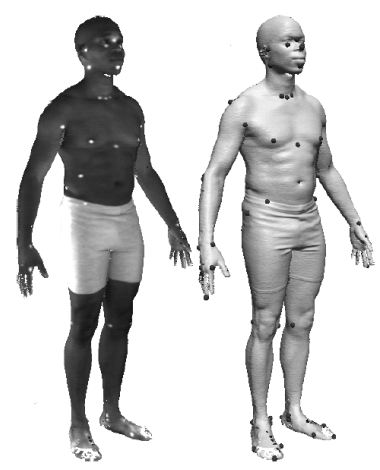

Fig. 2. Example of a scanned body shape from the CAESAR database

Such a representation ideally requires embedding different levels of knowledge:

- Geometric: What is the mesh resolution of the Virtual Human shape?

- Animation: What is the level of articulation of the Virtual Human model and is it able to perform a specific given sequence of motion?

- Morphological: What is the size, gender, or weight of a Virtual Human model?

- Behavioral: What are the skills, the mental state or the mood of a Virtual Human model?

These levels are intricately related: the size of a Virtual Human can be computed from the 3D shape of the body; the mental state will influence the gestures and motion that the Virtual Human can perform and the way it will perform them.

Current 3D search engines such as the Princeton 3D models search engine [5-8], are based on a few geometric metadata and 2D/3D shapes similarity and they are not able to process Virtual Human models deeper than at the geometric level [9]. There exists an obvious need to represent and store a Virtual Human model as a virtual personification of Humans. This representation should include the different layers of knowledge and semantic information so that every Virtual Human model can be efficiently searched and shared for populating Virtual Environments with avatars or autonomous characters.

\footnotetext{
${ }^{1}$ http://en.wikipedia.org/wiki/Anthropomorphic

${ }^{2}$ http://en.wikipedia.org/wiki/Personification
} 
Search is commonly performed using keywords or specific values of fields in the databases. This way of searching is very limited for complex multimedia objects such as Virtual Humans. For improving it we propose to use Ontologies, the technology used in the semantic web. The most complete language to express semantics is Ontology Web Language (OWL) [10]. This language is created upon Resource Definition Framework (RDF) [11]. OWL is a more expressive language than RFD, it provides relations between concepts in a logic way. Therefore it is possible, if implemented in a search engine, to make queries in natural language with a detailed criterion. In the sections 2 and 3 of this paper, we present the principal considerations for creating virtual individuals and some existing standards and good practice within these representations. In section 4 we present the structure of the proposed ontology. Using this ontology, we present a usage scenario that creates an individual from a semantic description is presented in section 5. Finally the conclusions and future work are presented in section 6 .

\section{Virtual Human Personification}

The personification process [12] in virtual humans includes mainly 3 stages:

\subsection{Physical Personification}

This aspect is related to the visual appearance of the Virtual Humans. We can consider 2 levels. The primary one that is mandatory regarding the human body itself and the secondary one that is optional regarding the

\subsubsection{Primary Physical Personification}

This personification step is dedicated to the modeling of 3D shape of the body. Although any 3D modeling software would provide the basic functionalities to create a $3 \mathrm{D}$ human body shape, new methods have been recently investigated in order to provide more intuitive approaches based on: parameterized human models; fitting predefined template or existing knowledge [13-17]. The scanning technology allows improving these new approaches by providing large amount of real data [2, 3]. Template-based method can be therefore improved by using statistical analysis methods to optimize the parameterization and control of the template model.

\subsubsection{Secondary Physical Personification}

This step is dedicated to an optional personification step, which consists in attaching accessories and artifacts (explicit hair, clothes, tools, jewels...) to the body shape. Accessories and artifacts are 3D shapes that are attached to some specific location of the body shape using landmarks.

Both categories refer to $3 \mathrm{D}$ geometry and share common characteristics. These characteristics refer those of any 3D shape; they can be: number of vertex, number of edges, a classification (mesh, contour, curve, etc), scale factor, material, texture, etc. Accessories and artifacts are connected to the body shape using landmarks that are located on the body surface. These landmarks are mainly 3D points or eventually 3D curves. 


\subsection{Expressional Personification}

Here we consider all aspects to give movement to a shape. As mentioned before for animating a character there is an implementation of articulation (skeletal structure) that drives the shape. As a result we have animation values to give to the articulations and shape deformation (skin binding). Shape deformation may be attached to the shape, or inside an algorithm. To produce animation values exist many techniques, we can split them in motion capture and key frame [18]:

\subsubsection{Motion Capture}

This animation technique is made with recorded movements of a real person using sensors in his body. This technique is more natural, but it is not easy to modify it; normally it is attached to a specific anatomy, and a lot retargeting work has to be made for reusing it.

\subsubsection{Keyframe Animation}

It is mainly defined as important frames that record the values of articulations, and the computer calculates with an interpolator the frames in between to produce the animation. This kind of animation can use other techniques as Inverse kinematics or dynamics. Its advantage is that one has high control of the animation but they are not realistic.

\subsection{Logical and Emotional Personification}

This is an open issue in research, where the results are the implementation of different kind of models to simulate behaviors [19, 20]. This model depends on the level of autonomy of the character. While less level of autonomy more complex is the model. Most of the created models are for completely autonomous characters. However those models have some characteristics in common, like the use of psychological models to represent affect (personality, emotion, mood, etc). It is important the representation of this individual characteristics, because they can be used to give expressions in animation, like happiness, anger, tiredness, etc. In fact some emotions are identified to drive synthetic animations [21].

We have mentioned the process that is followed in the creation of individual virtual humans. In the next section we will translate this process in terms of computational representation in a common practice.

\section{Virtual Human Representations}

Existing representations for Virtual Humans can be classified into two main categories: physical personification representations and anthropomorphic representations. The most developed is dedicated to physical representation and it is principally intended to describe the body shape, and control with joint motions or key frame animations. The other category is dedicated to provide higher level of personification with human-like attributes such as anthropometric or personality descriptors. Many of them are oriented to offer some kind of scripting animation language that allows defining expressive animations (i.e. animations that depicts 
emotions and behavior). None of these representations offers a common ground to integrate and cover all the aspects related to the personification of a Virtual Human models.

\subsection{Physical Personification Representation}

Most of these models are focused towards the representation and storage of:

- One or more 3D shapes that represent the outer shape of the body,

- Control animation structures, which include mainly an articulated control skeleton for the body.

- Skin binding information, which defines the way skin meshes are controlled and deformed according to skeleton motions.
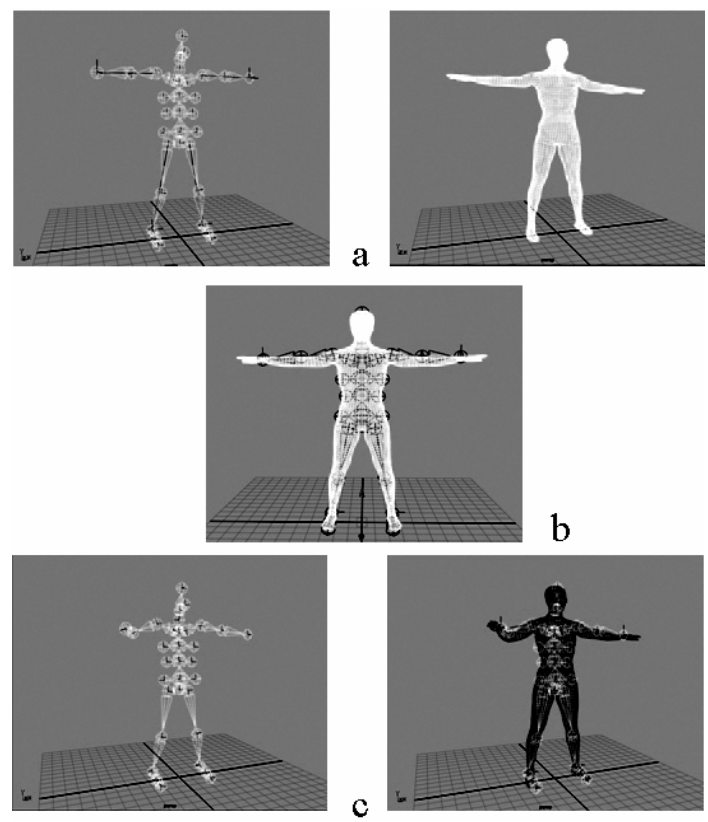

Fig. 3. (a) Skeleton (left) and skin modeling (right), (b) skin binding to the skeleton, (c) skeleton motion (left) and skin deformation (right)

These models are limited to the representation of the physical and expressional personification levels. They provide a low level of personification and ensure that the Virtual Human model look and move similarly to a real one. The main issue is to model an articulated skeleton that is able to reproduce as close as possible the degrees of freedom of the anatomical skeleton and to keep the 3D skin realistically adjusted around the skeleton for any posture with Skeleton Driven Deformation [22, 23] (Fig. 3).

The best way to share this physical representation is through standards. In computer graphics exists the standardization for the animation structure in H-Anim [24] standard. This structure mainly represents a simplified human skeleton with 
some specifications of the name, number and components of human articulations, as presented in the figure 4 . This standard has been used or could be used in many 3D exchange formats like MPEG-4 [25], VRML/X3D [26], Collada [27], CAL3D [28], $\mathrm{BVH}$ [29], etc. It provides an articulated structure with standard nomenclature and best practice. A typical example of the benefit of such standards is that an animation sequence can be shared among different 3D characters if they use H-Anim standard as animation control structure.

Still these formats can address much more detailed information of Virtual Human, like the level of articulation, level of detail, joint limit, joint coupling, etc. This kind of information is impossible to know at first instance, and they are important at the time of animating. MPEG-4 is also considering the face and facial animation and provides a scheme to control facial expressions based on control points. Facial animation in MPEG-4 also provides premises for higher level representation by taking into account six basic emotions (happiness, sadness, surprise, fear, anger and disgust).

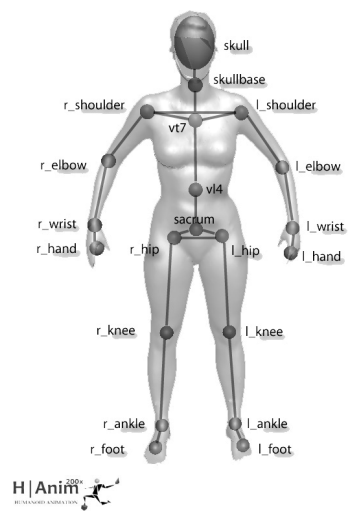

Fig. 4. H-ANIM standard representation or articulated Virtual Human

\subsection{Anthropomorphic Representations}

This type of representation allows addressing higher levels of knowledge, mainly morphology and behavior, which complete the personification of the Virtual Human models. A typical example of such representation model is the Human Markup Language. These representations are either too much targeted for a specific type of applications or too much abstract regarding the physical representation aspect.

\subsubsection{Multi-purposes Representations}

The Human Markup Language is an XML based representation proposed by the OASIS Human Markup Technical Committee. According to the Human Markup Language Primary Base Specification 1.0 [30, 31], the scope of the Human Markup Language is quite broad as it targets applications such as enabling real-time animated behaviors for $3 \mathrm{D}$ representations of humans to enhancing diplomatic communication with translation services and/or applications capable of making provisions for cultural practices. The Human Markup Language is expected to describe a fundamental set of 
characteristics of human entities and human activities as they occur in digital information systems. All these characteristics can be applied to represent artificial humans as well as real humans.

The Human ML basically includes simple types, such as age, gender or physical descriptors (weight, hair color, eye color...) and complex types such as Address, Human artifact (clothes, jewels...), Belief, Human Communication Channel, Community as an Abstract Human Organization, Human Culture, Human Emotion, Geolocator, Haptic (defined as the strength, location, and body part used in a touching behavior), Human Intent, Kinesic (Human Movements), Human Personality Type...

\subsubsection{Anthropometric Representations}

For representations such as the one proposed in [32], the target is CAD-CAM simulations involving human activities. The objective is to be able to query an inhabited Virtual Environment for controlling and analyzing CAD-CAM simulations. The proposal for representing Virtual Humans is focused towards the anthropometric attributes. It considers 1) human information: gender, stature, age, mass, joints, clothing, nationality...; 2) human's state: orientation, location, direction of motion, posture, joint angle... It also takes into account clothing, objects and environment.

\subsubsection{Behavioral Representations}

Other specific representations have been proposed with a more focused scope of application. Most of them are more scripting languages dedicated to represent animation of Virtual Human than representing the Virtual Human itself. The Character Mark-up Language (CML) is an XML-based scripting language proposed to link available engines for generating and controlling believable behavior of animated agents with the corresponding animated representations [33]. The Avatar Markup Language (AML), also based on XML, aims at encapsulating the Text to Speech, Facial Animation and Body Animation in a unified manner with appropriate synchronization [34]. The Emotion Annotation and Representation Language (EARL) [35] is focused in the behavioral aspects Emotion-oriented (or "affective") computing. They provide an annotation of emotions like intensity, variation, confidence, etc.; with the goal of interpretation and generation of behaviors.

Those XML languages offer a description for the representation of the concepts; they are adequate for annotation, and to provide a standardization of terms. According to the semantic stack [36] the next step is to define an ontology to provide a semantic meaning of the content.

\section{Ontological Representation of Virtual Humans}

Once we have a data structure and data concepts we can specify the relation of concepts to build the formalization of the knowledge. A diagram of the ontology is presented in the Figure 5. The concepts that are circled are subclasses of the concept resource (in the bottom of the diagram). Resource is the content that the user gets when searching for something. Resources have properties like file info (extension, size, URL), version, Author, creation date, etc. Some of the resources can be found in separated files or more than one in the same file. For the case of behavior controller the user can get an algorithm. 
Each shape representation we identified in the previous sections is represented as a concept; they are described in the ontology as follows:

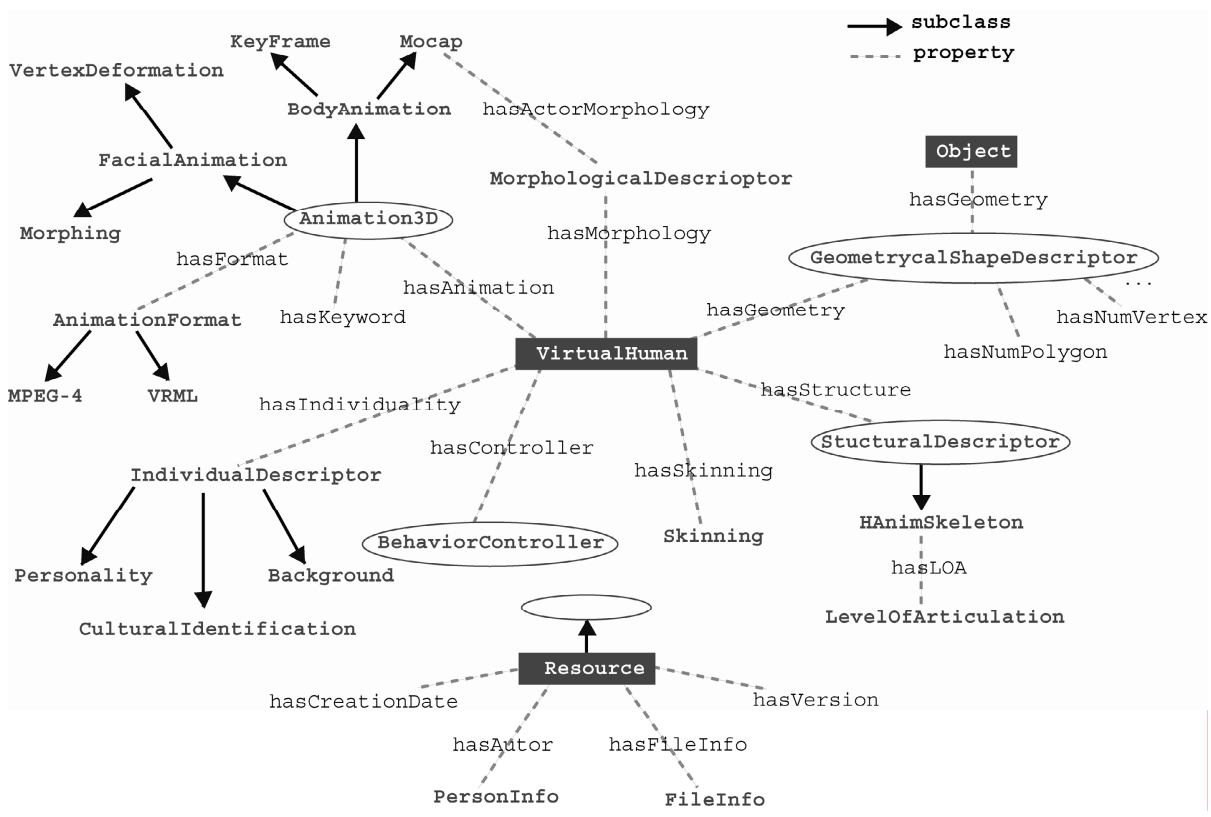

Fig. 5. Ontological representation of a Virtual Human

Geometry (hasGeometry, hasObject, hasGarment): The geometry is the physical visual representation of the Virtual Human and his two aspects, primary: body shape, and secondary: accessories, garments, etc. Therefore, we can create a general description for any kind of geometry shape and heritage its properties to specialized subclasses, specifically for Virtual Humans or objects. This common class contains the geometric properties: number of vertex, edges, scale, material and texture. The Virtual Human has a Geometrical Representation where we can know the kind of geometry it has (e.g. Surface mesh).

Animation (hasStructure, hasAnimation): The animation of Virtual Human can be for face or body. Each one has a format defined (MPEG-4, VRML, etc.), The way of animating each other is different, and it is possible to specialize the content of a face animation; for example if we have a happiness expression we can classify the animation values [37]. Body animation can be classified in KeyFrame or MoCap (Fig. 6). In order that an animation can take place, the character model should share the same kind of structure as the animation. This structure is defined in the Structural Descriptor (Fig. 7). We have based this structure in the H-Anim standard, which in fact is adopted in most of the geometry and animation file formats; however, any 


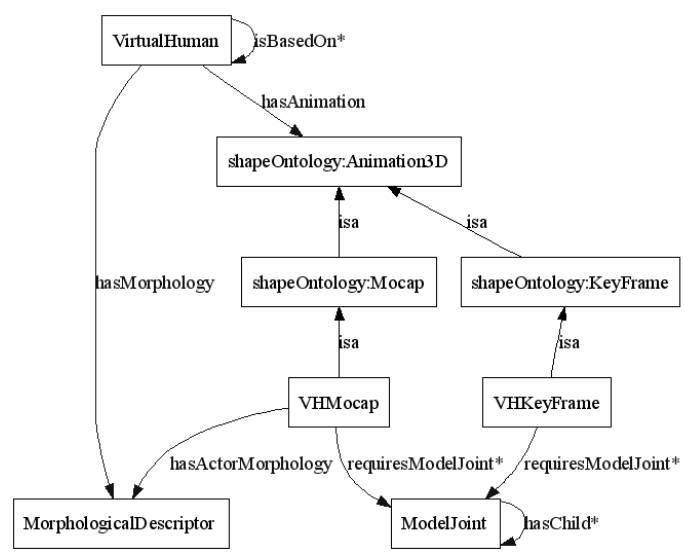

Fig. 6. Overview of the animation descriptor in the ontology

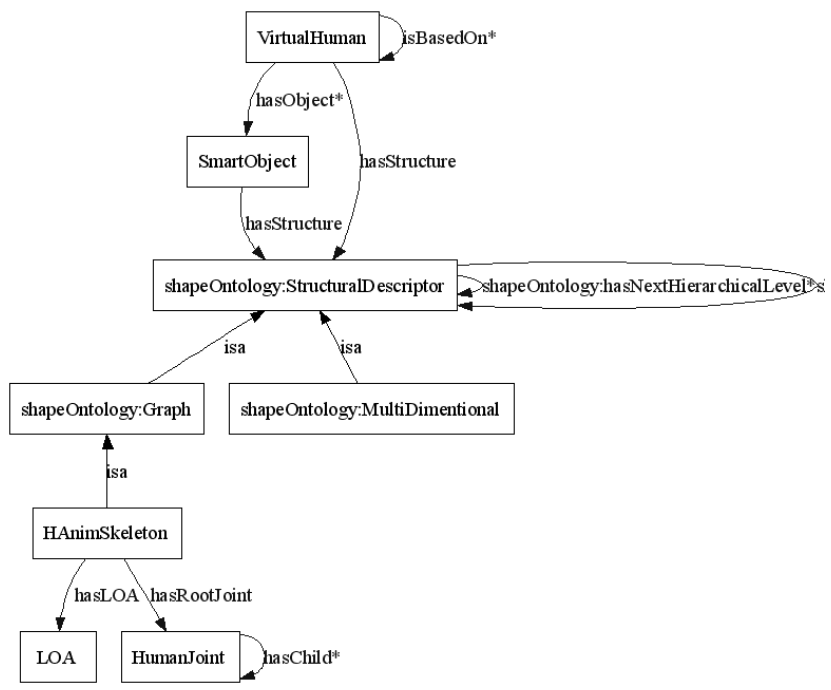

Fig. 7. Overview of the animation descriptor in the ontology

other structures can be also represented. The Motion Capture animation has also some special properties such as the morphology of the actor. This can be useful to predict and support the work one has to do in order to retarget an animation for a model with a different morphology.

Morphology (hasMorphology): Morphological Descriptor (Fig. 8) contains the information like: Age, weight, height, gender. This information is used to describe the Virtual Human body shape as people are used to do it for real human beings. 


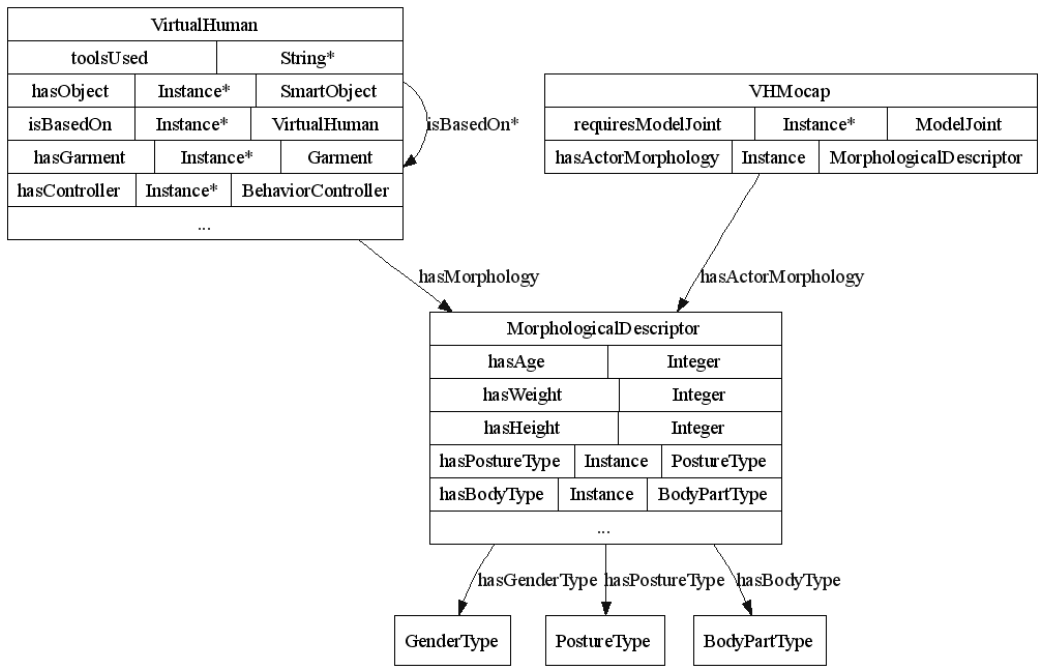

Fig. 8. Overview of the morphology descriptor in the ontology

Behavior (hasBehavior, hasIndividuality): Individual Descriptor (Fig. 9) and Behavior controller are for describing the behavior. The Individual descriptor contains the constant definition of the behavior of the Virtual Individual like his personality or cultural identification, background. The behavior controllers are algorithms that drive the behavior of the character considering the emotional state and its individuality.

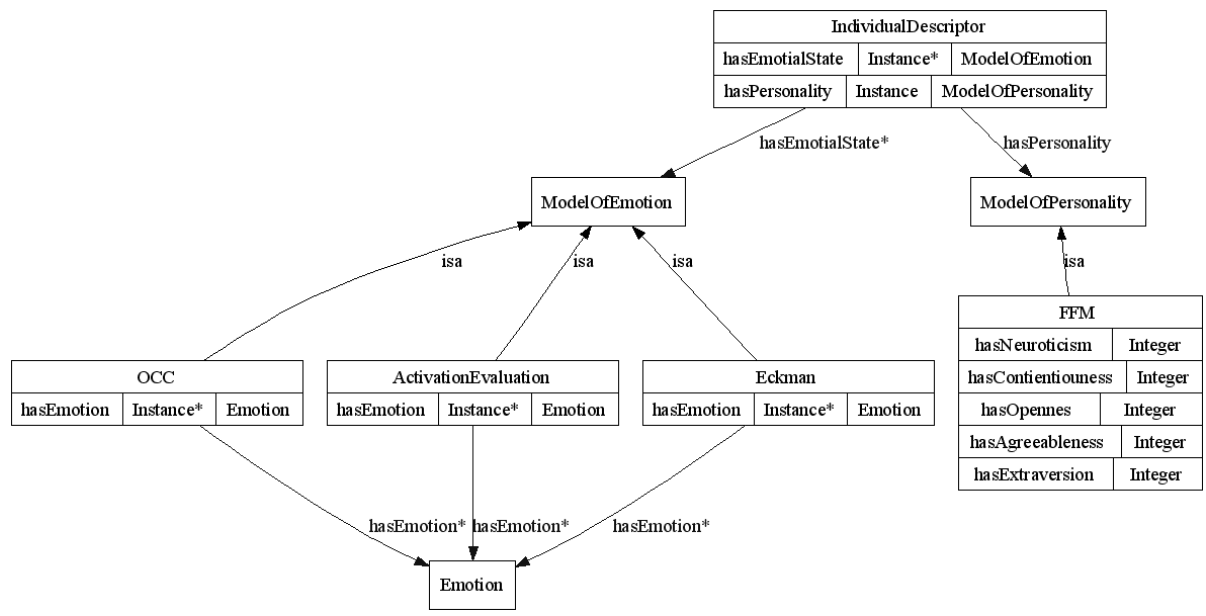

Fig. 9. Overview of the individuality descriptor in the ontology

This is a simplified version of the ontology with the most important definitions we have explained in this paper; however much more information has been considered, for example the structure of H-Anim (joints and segments), the animation formats of 
MPEG-4 (FAP, BAP), etc. Finally we can see in the diagram that there is a lot of information around one concept, the Virtual Human. There are physical concepts and abstract concepts that give the meaning to the Virtual Human. In the next section we will present a case when a user wants to build its own virtual character from scratch by searching the desired components in the ontology.

\section{Create a Virtual Individual}

To give an example of the creation of a virtual individual we present the process we may follow for playing a specific type of motion animation sequence. Here we have used Protégé [38] to create the instances of some of our resources. Also in Protégé with the nRQL [39] plug-in we search for the components we want.

\section{SEARCH 1}

Find: Animation 3d has Keyword: dance has Actror Morphology: has Gender:female

RESULT:

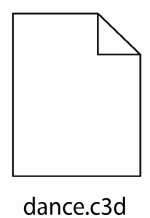

complementary information extracted with the file:

has Actror Morphology: has Heigth: 1.70

\section{SEARCH 2}

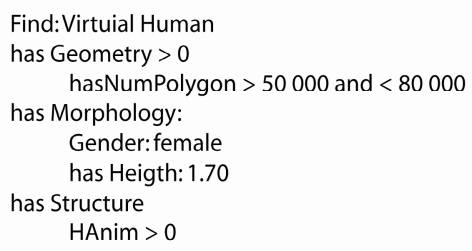

RESULT:
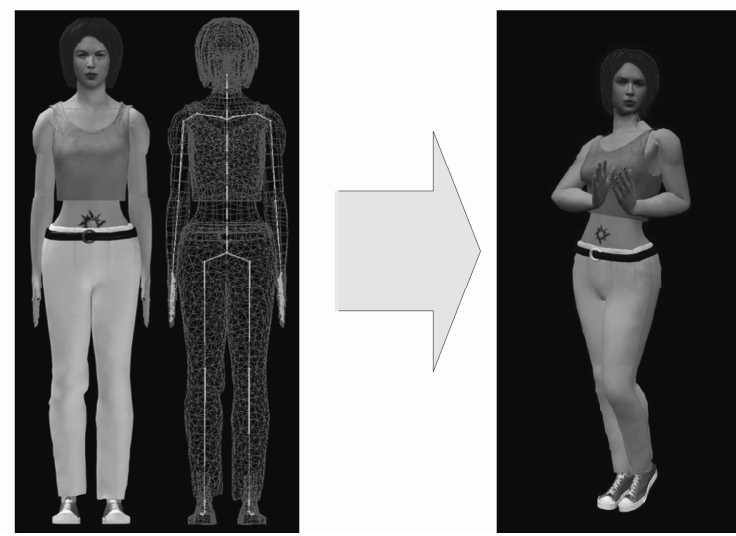

Fig. 10. Example of the creation of virtual individual

This example is illustrated in the figure 10. Let's say that our designer, Mireille, wants an animation of a woman dancing; she looks in the ontology for animations of women that have the keyword dancing. She found one in C3D [40] format which can be open it in Motion Builder [41]; there she makes a retargeting of the MoCap animation to the $\mathrm{H}$-anim skeleton, and she exports the animation to 3DMax [42]. There, she can export the animation in our proprietary format WRK, using a home made plug-in, this format uses $\mathrm{H}$-Anim standard. She found that the mocap animation is made for a woman of $1.70 \mathrm{~m}$. Therefore, she needs to search for a woman with a 
height of $1.70 \mathrm{~m}$, with a structure of H-Anim, and also with a number of polygons between $50 \mathrm{k}$ and $80 \mathrm{k}$, because that is the range we can load models and animate in our viewer without problems. She found a model in Collada format that can be opened in 3DMax, and exported in the format we use in the lab. Finally we can load both model in a 3D sequence viewer and play the animation.

This process can take 30 minutes in the best case, and much more depending on how "clean" are files that the designer got. We have noticed the benefit it could imply if we simplify the work using an optimized search like the one above. Currently a designer would first need to locate and identify the dancing sequence by playing each of the available animation sequences, and then to check if the dancing sequence fits the selected human model. They may have to do a lot of retargeting job because the scale of the model it was made the animation is not the same as the model we want to apply to, and much more other issues.

More complicated search can take more time in the adaptation. For example, when one can use more complex elements in a scenario where the user search for a behavioral algorithm, and with the information annotated in it (e.g. what kind of character the algorithm can be applied?), the user can search for the missing elements depending on the algorithm's requirements.

\section{Conclusions and Future Work}

In this paper we have proposed an approach to share 3D content, specifically for the creation of virtual individuals. We have considered 3 essential elements that are involved in the creation of these characters: geometry, animation, morphology and behavior. We have presented an ontology that makes explicit the relation of the elements, and we have presented a scenario where the knowledge of the semantics of the elements can improve the creation of virtual characters. We encourage the use of standards to be able to achieve examples as the one proposed in section 5 , because the process of sharing models among designers or institutions is almost impossible because of the lack of the use standards and semantics. In the context of standardization the knowledge we may look at the existing XML proposals mentioned in the section 3.2 to ensure that other contributions that go in the same direction are compatible. In that way the standardization of the knowledge can take place.

Within our future work, we consider the implementation of a searching application for this ontology. We need a search engine capable of making reasoning. This is taking place within the AIM@SHAPE network [43, 44]. Moreover, a difficult issue that needs to be faced is at the moment of extraction of metadata from resources to populate the ontology. However, the extraction of the metadata form the most of the 3D files can be made with applications to automate the process.

\section{Acknowledgements}

This research has been funded by the EC and the Swiss Federal Office for Education and Science in the framework of the European Networks of Excellence IST-AIM@SHAPE (http://www.aim-atshape.net) and IST-HUMAINE (http://emotionresearch.net). 


\section{References}

1. Station Exchange. http://stationexchange.station.sony.com/.

2. B. Allen, B. Curless, and Z. Popovic. The space of human body shapes: reconstruction and parameterization from range scans. in SIGGRAPH '03. 2003.

3. H. Seo and N. Magnenat-Thalmann, An Example-Based Approach to Human Body Manipulation, in Graphical Models, A. Press, Editor. 2004. p. 1-23.

4. S. Kshirsagar and N. Magnenat-Thalmann. A Multilayer Personality Model. in 2nd International Symposium on Smart Graphics. 2002.

5. Princeton3D, models search engine. $\mathrm{http}: / /$ shape.cs.princeton.edu/search.html.

6. O. IV. http://ship.nime.ac.jp/ motofumi/Ogden/.

7. D. Search. http://3d-search.iti.gr/3DSearch.

8. C.s. Characteristics, Content-based Classification of 3D-models. http://merkur01.inf.unikonstanz.de/CCCC/.

9. R.C. Veltkamp and J.W.H. Tangelder, eds. Content Based 3D Shape Retrieval. Encyclopedia of Multimedia. 2006, Springer Borko Furht (Ed.).

10. OWL, Web Ontology Language Overview. http://www.w3.org/TR/owl-features/.

11. RDF, Resource Description Framework. http://www.w3.org/RDF/.

12. N. Magnenat-Thalmann and D. Thalmann, Handbook of Virtual Humans, ed. Wiley. 2004.

13. C. C. and L. Wang, Parameterization and parametric design of mannequins, in ComputerAided Design. 2005. p. 83-98.

14. W. Lee, J. Gu, and N. Magnenat-Thalmann, Generating animatable three-dimensional virtual humans from photographs. Computer Graphic Forum 2000. 19(3): p. 1-10.

15. K.-M. Tong, K.-C. Hui, C. C., and L. Wang. Mesh Fitting Based 3D Character Modeling. in Edutainment 2006. 2006.

16. P.P.J. Sloan, C.F. Rose, and M.F. Cohen. Shape by example. in Symposium on Interactive 3D Graphics. 2001.

17. MAKEHUMAN. http://www.dedalo3d.com/index.php?filename=SXCOL/makehuman/ abstract.html.

18. R. Parent, Computer Animation: Algorithms and Techniques, ed. Morgan-Kaufmann. 2001, San Francisco.

19. E.d. Sevin and D. Thalmann. An Affective Model of Action Selection for Virtual Humans. in Agents that Want and Like: Motivational and Emotional Roots of Cognition and Action symposium at the Artificial Intelligence and Social Behaviors. 2005. Hatfield, England.

20. T. Conde and D. Thalmann. Autonomous Virtual Agents Learning a Cognitive Model and Evolving. in Intelligent Virtual Agents, Lecture Notes in Computer Science. 2005: Springer-Verlag

21. P. Eckman, Facial expression and emotion, in Am Psychologist. 1993. p. 384-392.

22. J.P. Lewis, M. Cordner, and N. Fong. Pose Space Deformations: A Unified Approach to Shape Interpolation and Skeleton-Driven Deformation. in SIGGRAPH '00 2000: AddisonWesley.

23. B. Allen, B. Curless, and Z. Popovic. Articulated body deformation from range scan data. in SIGGRAPH 2002. 2002.

24. H-anim., The humanoid animation working group. http://www.h-anim.org.

25. MPEG-4, Overview of the MPEG-4 standard. http://www.chiariglione.org/mpeg/ standards/mpeg-4/mpeg-4.htm.

26. Web 3D consortium. http://www.web3d.org/.

27. COLLADA, Asset Exchange Schema for Interactive 3D. http://www.khronos.org/collada/.

28. CAL3D. http://home.gna.org/cal3d/. 
29. Biovision, $B V H$. http://www.cs.wisc.edu/graphics/Courses/cs-838-1999/Jeff/BVH.html.

30. J. Landrum and S.C.d. Ram. Human Markup Language [HumanML]: Humanness Content and Sharing across Perspective Shifts. in CIDOC CRM Special Interest GroupConference. 2003. Washington, D.C.

31. J. Peltz, HumanML: The Vision, Jay Peltz, in DM Direct Newsletter. 2005 http://www. dmreview.com/article_sub.cfm?articleID $=1033534$.

32. J.D. Ianni. Standardizing Human Model Queries. in Digital Human Modeling For Design And Engineering Conference And Exhibition. 2001. Arlington, VA, USA.

33. Y. Arafa and A. Mamdani. Scripting Embodied Agents Behavior with CML: Character Markup Language. in International Conference on Intelligent User Interfaces. 2003. New York: ACM Press.

34. S. Kshirsagar, A.Guye-Vuilleme, K. Kamyab, N. Magnenat-Thalmann, D. Thalmann, and E. Mamdani. Avatar Markup Language. in 8th Eurographics Workshop on Virtual Environments. 2002.

35. M. Schröder, H. Pirker, and M. Lamolle. First suggestions for an Emotion Annotation and Representation Language. in Language Resources and Evaluation. 2006. Genoa, Italy.

36. W3C, Semantic stack architecture. http://www.w3.org/2000/Talks/1206-xml2ktbl/slide10-0.html.

37. F. Vexo A. Garcia-Rojas, D. Thalmann, A. Raouzaiou, K.Karpouzis, S. Kollias, L. Moccozet and N. Magnenat-Thalmann., Emotional Face Expression Profiles Supported by Virtual Human Ontology. Computer Animation and Virtual Worlds Journal, 2006. 17(Special Issue): p. 259-269.

38. Protégé, Stanford medical informatics. $2005 \mathrm{http} / / /$ protege.stanford.edu/index.html.

39. nRQL, New racer query language (nrql) interface to owl ntologies in Protégé. http://www. cs.concordia.ca/ k_bhoopa/nrql.html.

40. C3D, Coordinate 3D. http://www.c3d.org/.

41. Ronan Boulic, Ramon Mas, and Daniel Thalmann, A robust approach for the control of the center of mass with inverse kinetics. Computers \& Graphics, 1996. 20(5): p. 693-701.

42. Autodesk 3ds Max. http://usa.autodesk.com/adsk/servlet/index?id=5659302\&siteID=123112.

43. R. Albertoni, L. Papaleo, M. Pitikakis, F. Robbiano, M. Spagnuolo, and G. Vasilakis, Ontology-based Searching Framework for Digital Shapes, in Applications of Semantic Web II (SWWS) L.N.i.C. Science, Editor. 2005, Springer-Verlag GmbH. p. 896-905.

44. George Vasilakis, Marios Pitikakis, Manolis Vavalis, and Catherine Houstis. A Semantic Based Search Engine for 3D Shapes: Design and Early Prototype Implementation. in 2nd European Workshop on the Integration of Knowledge, Semantic and Digital Media Technologies (EWIMT2005). 2005. http://www.iee.org/oncomms/pn/visualinformation/ ewimt2005_0067.pdf. 